\title{
Hepatic TRAP80 selectively regulates lipogenic activity of liver $\mathrm{X}$ receptor
}

\author{
Geun Hyang Kim, ${ }^{1,2}$ Gyun-Sik Oh, ${ }^{1,2}$ Jin Yoon, ${ }^{1,2}$ Gang Gu Lee, ${ }^{1,2}$ Ki-Up Lee, ${ }^{3}$ and Seung-Whan Kim ${ }^{1,2}$ \\ ${ }^{1}$ Department of Pharmacology, Asan Medical Center, ${ }^{2}$ Bio-medical Institute of Technology, and ${ }^{3}$ Department of Internal Medicine, Asan Medical Center, \\ University of Ulsan College of Medicine, Seoul, Republic of Korea.
}

\begin{abstract}
Inflammation in response to excess low-density lipoproteins in the blood is an important driver of atherosclerosis development. Due to its ability to enhance ATP-binding cassette A1-dependent (ABCA1-dependent) reverse cholesterol transport (RCT), liver X receptor (LXR) is an attractive target for the treatment of atherosclerosis. However, LXR also upregulates the expression of sterol regulatory element-binding protein 1c (SREBP-1c), leading to increased hepatic triglyceride synthesis, an independent risk factor for atherosclerosis. Here, we developed a strategy to separate the favorable and unfavorable effects of LXR by exploiting the specificity of the coactivator thyroid hormone receptor-associated protein 80 (TRAP80). Using human hepatic cell lines, we determined that TRAP80 selectively promotes the transcription of SREBP-1C but not ABCA1. Adenovirus-mediated expression of shTRAP80 inhibited LXR-dependent SREBP-1c expression and RNA polymerase II recruitment to the LXR responsive element (LXRE) of SREBP-1c, but not to the LXRE of ABCA1. In murine models, liver-specific knockdown of TRAP80 ameliorated liver steatosis and hypertriglyceridemia induced by LXR activation and maintained RCT stimulation by the LXR ligand. Together, these data indicate that TRAP80 is a selective regulator of hepatic lipogenesis and is required for LXR-dependent SREBP-1c activation. Moreover, targeting the interaction between TRAP80 and LXR should facilitate the development of potential LXR agonists that effectively prevent atherosclerosis.
\end{abstract}

\section{Introduction}

Liver X receptor (LXR) is a member of the nuclear receptor superfamily and acts as a cholesterol sensor to regulate the expression of several key proteins involved in lipid metabolism and inflammation $(1,2)$. Ligand-induced transactivation by nuclear receptors is a finely organized process that requires a variety of coactivators (3-5). Preferential functional association between particular nuclear receptors and coactivators may promote the specific regulation of individual genes under various conditions $(6,7)$. At present, a limited amount of information is available regarding coactivators of LXR and their mechanistic and physiological roles in LXR-mediated transactivation $(8,9)$.

Atherosclerosis is the leading cause of mortality in developed countries and is poised to become a worldwide health problem (10). LXR is a promising target for the treatment of atherosclerosis due to its ability to inhibit macrophage-driven inflammation and enhance ABCA1-dependent reverse cholesterol transport (RCT) (11-14). RCT is a pathway by which excess cellular cholesterol is transported from peripheral tissues to the liver for excretion in the bile and ultimately the feces, thereby helping to prevent atherosclerosis (15). Increased RCT mediated by ABCA1 upon treatment with the LXR ligands T0901317 or GW3965 contributes to an increase in serum HDL and a decrease in atherosclerosis $(16,17)$. Moreover, LXR exerts a further atheroprotective effect through inhibition of the macrophage inflammatory gene response (11,

Authorship note: Geun Hyang Kim and Gyun-Sik Oh contributed equally to this work. Conflict of interest: The authors have declared that no conflict of interest exists. Submitted: October 7, 2013; Accepted: October 30, 2014.

Reference information: / Clin Invest. 2015;125(1):183-193. doi:10.1172/JCI73615.
18). These favorable effects of LXR agonists may be therapeutic in patients with atherosclerotic disease. However, LXR activation is also associated with increased mRNA levels of $S R E B P-1 c$, the master regulator of de novo fatty acid biosynthesis, leading to elevated serum and liver triglyceride levels (19-21). To optimize the therapeutic benefits of LXR agonists, it will be necessary to inhibit the unfavorable effects of LXR on fatty acid metabolism.

This study was initiated to clarify the mechanism of LXRmediated transcription of metabolic genes, with particular focus on the details of the coactivator complex and a view toward modulating specific target gene expression. Here, we present 4 LXR $\alpha$-interacting coactivators and demonstrate that 1 of these LXR $\alpha$-interacting coactivators, TRAP80, is selectively recruited to the LXR responsive element (LXRE) of SREBP-1c, but not of ABCA1. TRAP80 knockdown by adenoviral shTRAP80 infection inhibited the transcription of lipogenic genes, including $S R E B P-1 c$, fatty acid synthase $(F A S)$, and stearoyl CoA desaturase 1 (SCD1), but not the transcription of RCT-related genes such as $A B C A 1$, $A B C G 5$, and $A B C G 8$. Furthermore, hepatic delivery of adenoviral shTRAP80 (Ad-shTRAP80) ameliorated hepatic steatosis induced by LXR-activated lipogenesis. Collectively, our data provide insight into the coactivator selectivity for transcriptional activation and suggest a strategy that could be exploited to amplify the beneficial effects of LXR agonists.

\section{Results}

Identification of TRAP8O and TRAP22O as direct LXR $\alpha$-interacting proteins. To isolate nuclear proteins as possible coactivators that interact independently or cooperatively with LXR $\alpha$, we incubated purified GST-fused LXR $\alpha$ ligand-binding domain (LBD) with 

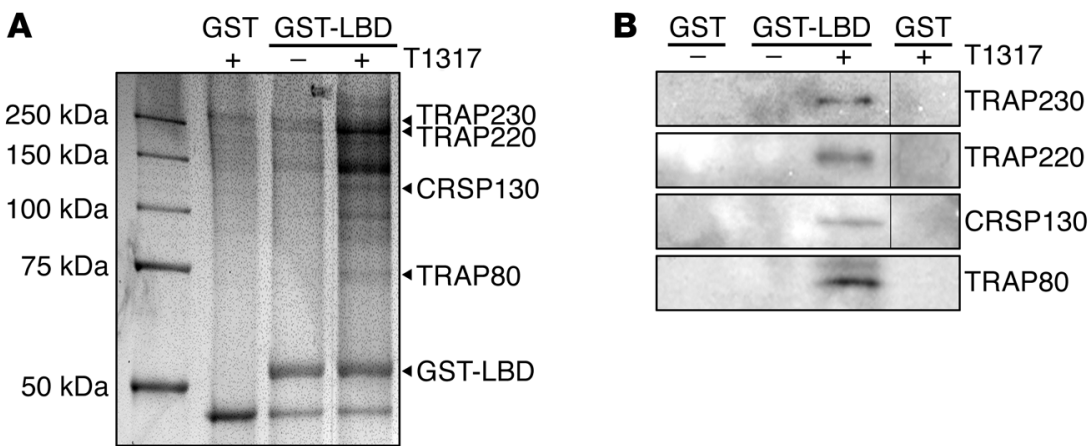

C

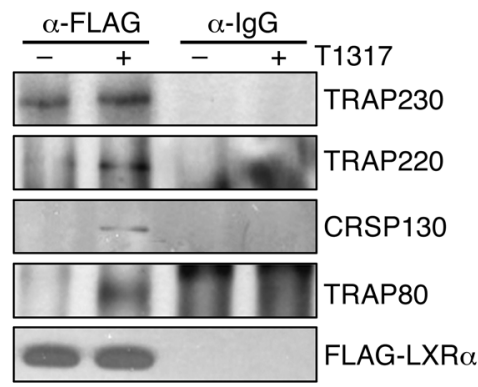

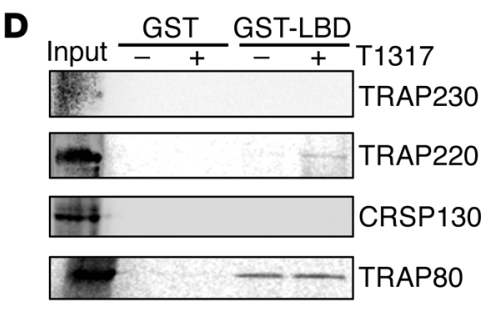

Figure 1. Purification and identification of proteins interacting with LXR $\alpha$ LBD. (A) Ligand-dependent interactions between LXR $\alpha$ LBD and several nuclear proteins from HepC2 cells. Immobilized CST-LXR $\alpha$ LBD was incubated with HepG2 nuclear extracts in the presence or absence of T0901317. Immobilized GST was used as a control protein in the presence of ligand. Arrowheads indicate $L X R \alpha$-interacting proteins analyzed by MALDI-TOF. (B) Identification of LXR $\alpha$ LBD interactors by immunoblot analysis. The thin black lines indicate that the lanes were run on the same gel but were noncontiguous. The TRAP80 blot was derived from samples run on a parallel gel. (C) Immunoblot analyses of proteins immunoprecipitated from FLAG-LXR $\alpha$-transfected HEK293T cells using anti-FLAG antibodies. (D) GST pull-down assays were used to evaluate the binding between GST-LXR $\alpha$ LBD and $\left[{ }^{35} \mathrm{~S}\right]$-methionine-labeled interactors. GST-LBD, CST-LXR $\alpha$ LBD; T1317, T0901317.
HepG2 nuclear extracts in the presence or absence of the LXR agonist T0901317 (Figure 1A and Supplemental Figure 1; supplemental material available online with this article; doi:10.1172/ JCI73615DS1). Compared with GST alone, 4 proteins bound specifically to GST-LXR $\alpha$ LBD in a ligand-dependent manner (Figure 1A). Using matrix-assisted laser desorption ionizationtime-of-flight (MALDI-TOF) analysis, we identified these LXR $\alpha$ LBD-interacting proteins as TRAP230 (ARC240 and MED12), TRAP220 (DRIP205, CRSP200, PBP, and MED1), CRSP130 (DRIP130, ARC130, SUR2, and MED23), and TRAP80 (DRIP80, ARC77, CRSP77, and MED17) (Supplemental Table 1 and refs. 22-26). We confirmed the identities of the GST-LXR $\alpha$ LBD-bound proteins by immunoblot analyses with specific antibodies (Figure 1B). To verify that these interactions took place inside the cells, total lysates from FLAG-LXR $\alpha$-transfected cells were immunoprecipitated with anti-FLAG antibodies and subsequently probed with antibodies against each interacting protein (Figure 1C). All 4 proteins interacted with $\mathrm{LXR} \alpha$ in a ligand-dependent manner in the cells. These results demonstrate that LXR $\alpha$ interacts with TRAP230, TRAP220, CRSP130, and TRAP80 via its LBD in a ligand-dependent manner.

Next, we examined whether any of these proteins bind directly to the LXR $\alpha$ LBD using in vitro-translated and $\left[{ }^{35} \mathrm{~S}\right]$-labeled interactors. Only 2 of the proteins, TRAP220 and TRAP80, interacted directly with the LXR $\alpha$ LBD (Figure 1D). Interestingly, $\left.{ }^{35} \mathrm{~S}\right]$-TRAP220 bound to GST-LXR $\alpha$ LBD in a ligand-dependent manner, whereas the $\left[{ }^{35} \mathrm{~S}\right]$-TRAP80 interaction with GST-LXR $\alpha$ LBD was ligand independent in a 2-component protein-protein interaction assay.

TRAP8O stimulates the transcriptional activity of the LXRE of SREBP-1c but not of the LXRE of ABCA1. To determine the functional relevance of the interaction of TRAP220 and TRAP80 with LXR $\alpha$, we performed cotransfection and luciferase reporter assays. Both TRAP220 and TRAP80 stimulated the transcrip- tional activity of LXR $\alpha$ on a synthetic LXRE promoter in a ligand-dependent manner (Figure 2A). We also examined their coactivator functions on 2 physiologically important natural promoters containing LXRE, specifically those of SREBP-1c and $A B C A 1$. We observed that TRAP220 enhanced liganddependent transactivation of both the SREBP-1C and ABCA1 promoters (Figure 2, B and D). By contrast, TRAP80 increased the transcriptional activity of the $S R E B P-1 c$ promoter, but not that of the $A B C A 1$ promoter (Figure 2, B and D). Importantly, neither TRAP220 nor TRAP80 stimulated the transcription of SREBP-1c and $A B C A 1$ promoters containing mutations in the LXRE, indicating that their coactivator function, at least in these cases, is specifically dependent on LXR recruitment to the LXRE (Figure 2, $\mathrm{C}$ and $\mathrm{E}$ ). We also examined this target gene-specific activity of TRAP80 using GW3965, a specific LXR ligand structurally unrelated to T0901317. Again, TRAP80 showed LXR-mediated selective activation of the SREBP-1c promoter by GW3965 in a dose-dependent manner (Figure $2 \mathrm{~F}$ ). Dose-dependent activation of the ABCA1 promoter by GW3965 was not affected by TRAP80 (Figure 2G). Meanwhile, we found that TRAP230 and CRSP130, the indirect interactors of $\mathrm{LXR} \alpha$, stimulated both $S R E B P-1 c$ and $A B C A 1$ LXRE promoters (Supplemental Figure 2).

In vivo transcription occurs on a nucleosomal template, i.e., chromatin. To investigate the recruitment of TRAP220 and TRAP80 in endogenous chromatin, we performed ChIP analyses of the Srebp-1c and Abca1 promoter regions (Figure 3A). Both TRAP220 and TRAP80 were recruited to the LXRE of the endogenous Srebp-1c promoter in response to treatment with T0901317 or GW3965 (Figure 3, B and D). By contrast, TRAP220 was recruited to the LXRE of the Abca1 promoter, but TRAP80 was not (Figure $3, \mathrm{C}$ and $\mathrm{E}$ ). We also investigated the recruitment of TRAP220 and TRAP80 under the condition of LXR $\alpha$ knockdown to confirm whether the recruitment of those coactivators is LXR $\alpha$ dependent. LXR $\alpha$-specific knockdown in mouse primary hepatocytes 

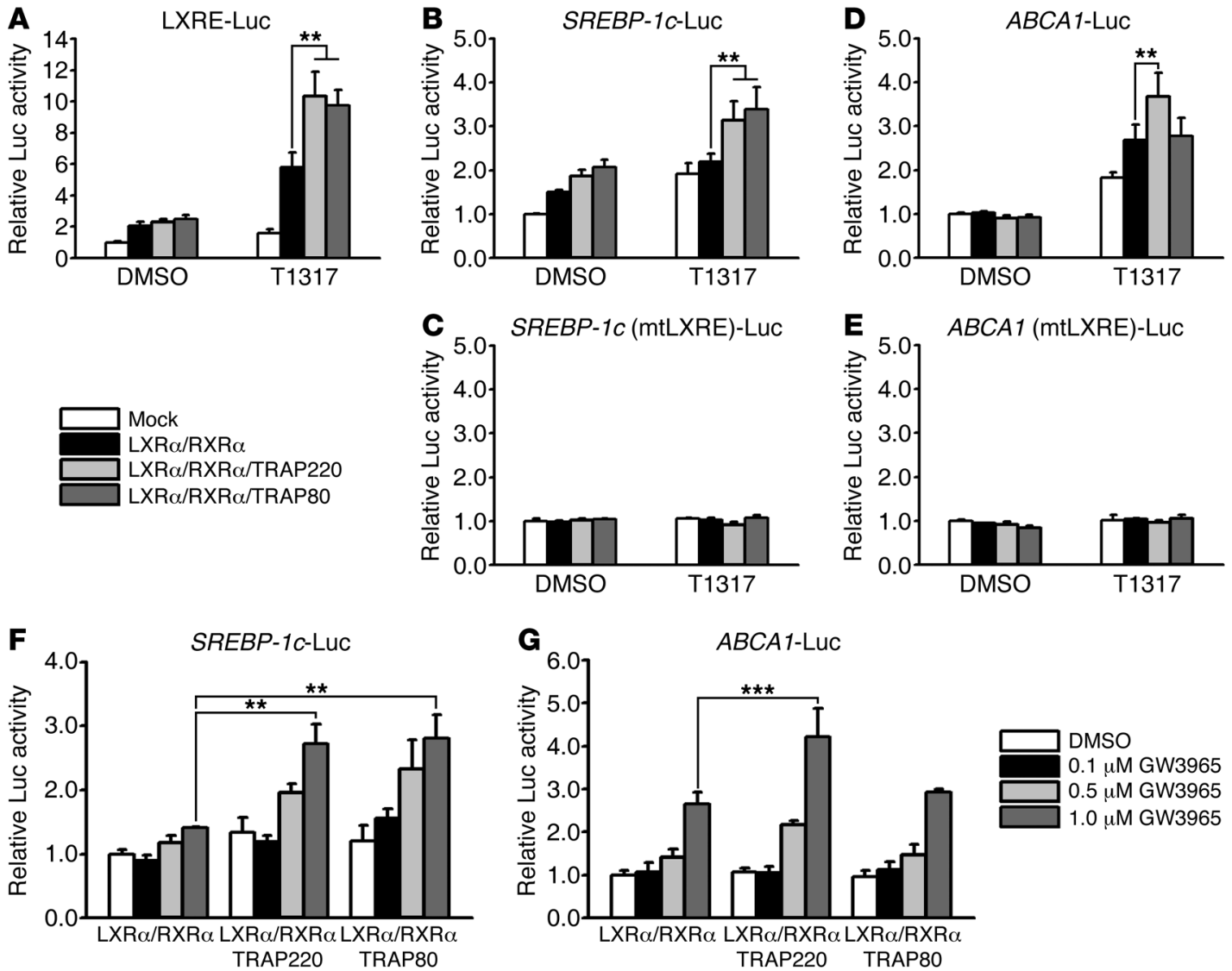

Figure 2. TRAP80 selectively stimulates SREBP-1c promoter activity in a ligand-dependent manner via LXRE. HepG2 cells were transfected with a luciferase reporter plasmid under the control of synthetic LXRE (A, $n=7)$, SREBP-1c WT promoter $(\mathbf{B}, n=9 ; \mathbf{F}, n=6), A B C A 1$ WT promoter (D, $n=10$; G, $n=6)$, SREBP-1c mutant promoter $(\mathbf{C}, n=6)$, or ABCA1 mutant promoter $(\mathbf{E}, n=5)$ with mutated DR-4 LXRE. T0901317 (A-E) or CW3965 (F and $\mathbf{G})$ was used to activate the LXRs. Data are presented as the mean \pm SEM. T1317, T0901317; Luc, luciferase; mt, mutant. ${ }^{* *} P<0.01$ and ${ }^{* *} P<0.001$, by 1-way ANOVA.

by siRNA was validated by quantitative reverse transcriptase PCR (qRT-PCR) (Supplemental Figure 3). As a result, the recruitment of both TRAP220 and TRAP80 to Srebp-1c LXRE was not stimulated by LXR ligands under LXR $\alpha$-knockdown conditions (Figure 3, B and D). We found that TRAP80 was not recruited to Abca1 LXRE, regardless of the expression of LXR $\alpha$ (Figure $3 \mathrm{E}$ ). Interestingly, we observed that the recruitment of TRAP220 to the Abca1 LXRE was increased by LXR ligands, even under LXR $\alpha$-knockdown conditions (Figure 3C). In conclusion, TRAP80 was selectively recruited in a ligand- and LXR $\alpha$-dependent manner to the Srebp-1c promoter, but not to the Abcal promoter.

TRAP8O regulates the recruitment of RNA polymerase II and histone modification of the LXRE region of Srebp-1c, but not that of Abcal. To address the consequences of the differential effects of TRAP80 on the Srebp-1c and Abca1 promoters, we introduced TRAP8O cDNA or Ad-shTRAP80 into the cells and determined the levels of Srebp-1c and Abca1 transcripts by qRT-PCR. TRAP80 overexpression significantly increased Srebp-1c mRNA expression, but did not affect Abca1 mRNA levels (Figure 4A). Consistent with this, Ad-shTRAP80 significantly suppressed the T0901317-dependent increase of Srebp-1c transcription, but did not affect Abca1 transcription (Figure 4B). Knockdown of TRAP80 using siRNA yielded results similar to those obtained with Ad-shTRAP80 (Supplemental Figure 4). To further inves- tigate the role of TRAP80 in Srebp-1c and Abca1 promoter activation, we performed ChIP assays using antibodies against RNA polymerase II ( $\mathrm{Pol}$ II) or acetyl-histone H4 (H4Kac). While Ad-shTRAP80 abolished the T0901317-dependent recruitment of Pol II to the LXRE of the Srebp-1c promoter, we found that recruitment of Pol II to the LXRE of the Abca1 promoter was not significantly altered (Figure $4 \mathrm{C}$ ). The recruitment of transcriptional regulators to the promoter chromatin is coordinated by epigenetic information encoded in covalent modifications of histone proteins (27). Histone acetylation is a central switch that allows interconversion between transcriptionally permissive or repressive chromatin states (28). We found that infection of mouse primary hepatocytes with Ad-shTRAP80 consistently decreased T0901317-dependent acetylation of histone $\mathrm{H} 4$ lysine (H4K) in the vicinity of the LXRE of the Srebp-1c promoter (Figure 4D). However, ligand-dependent acetylation of $\mathrm{H} 4 \mathrm{~K}$ near the LXRE of the Abca1 promoter was still significantly increased in the presence of shTRAP80, although this acetylation was slightly attenuated (Figure 4D). Meanwhile, we observed that LXR was recruited to the LXRE regions of the Srebp-1c and Abca1 genes in a ligand-dependent manner, regardless of TRAP80 expression (Figure 4E). Taken together, these results suggest that TRAP80 selectively stimulates SREBP-1c expression by Pol II recruitment and chromatin remodeling. 
A

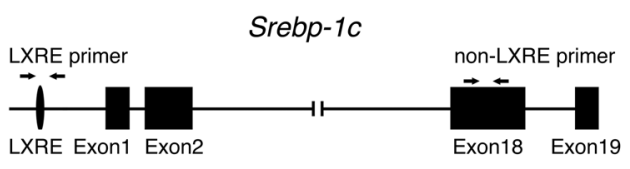

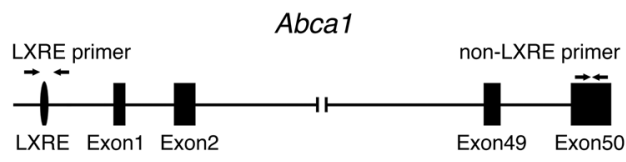
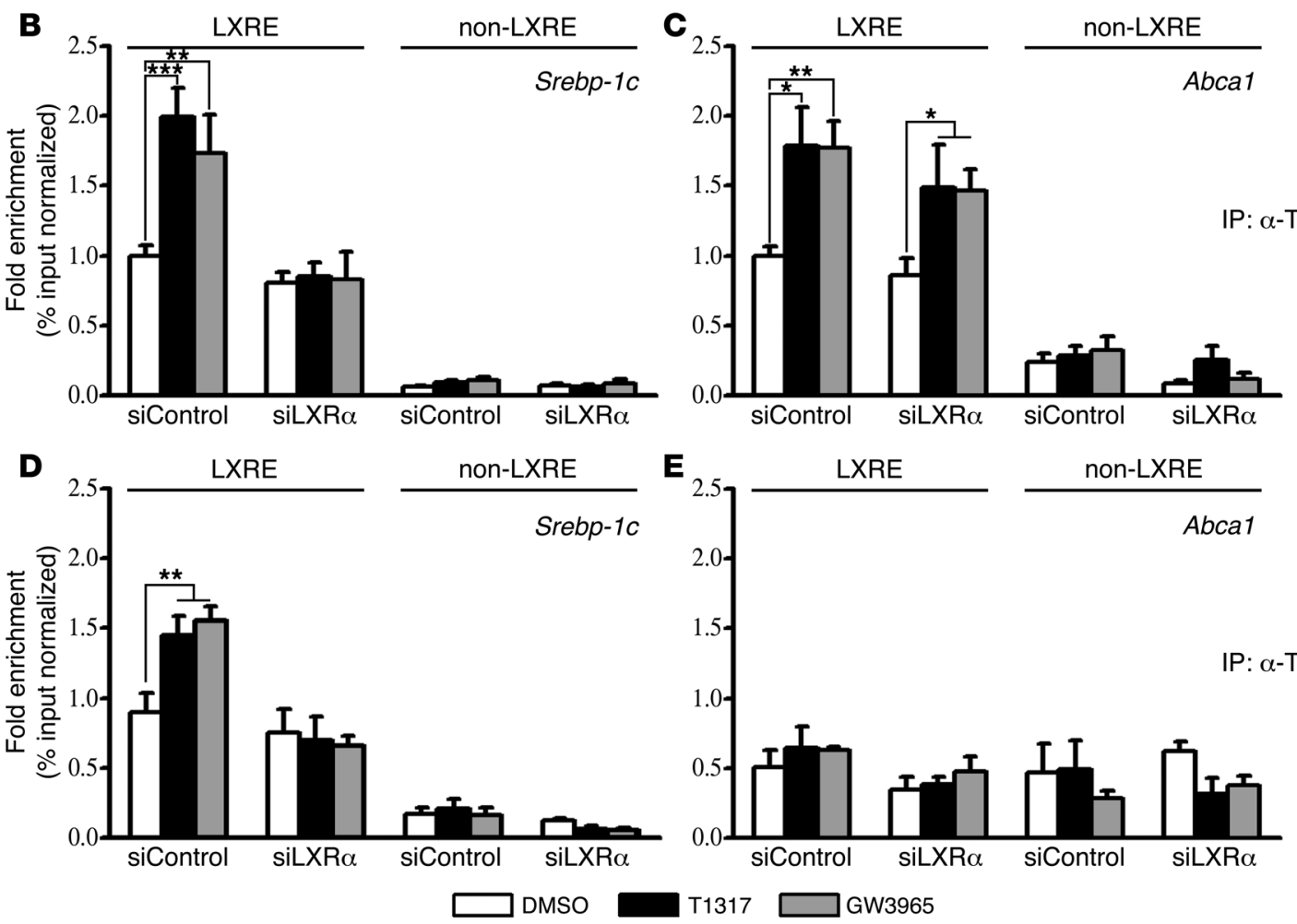

Figure 3. TRAP80 is selectively recruited to the LXRE of the Srebp-1c promoter. (A) Schematic diagram of primer locations used for the ChIP assay across the Srebp-1c or Abca1 genomic structure. (B-E) ChIP assays were performed with anti-TRAP220 (B and C) or anti-TRAP80 (D and E) antibodies in mouse primary hepatocytes following LXR $\alpha$ knockdown by siRNA (sc-38829). Recruitment of TRAP220 or TRAP80 to the LXREs of the Srebp-1c and Abca1 genes was determined by qPCR $(n=3)$. The level of recruitment is expressed as the fold enrichment relative to the recruitment of TRAP220 to the LXREs in the presence of DMSO alone. Data are presented as the mean \pm SEM. T1317, T0901317. ${ }^{*} P<0.05,{ }^{* *} P<0.01$, and ${ }^{* * *} P<0.001$, by 1 -way ANOVA.

Hepatic knockdown of TRAP8O represses LXR-dependent lipogenesis in the liver. To investigate the physiological effects of TRAP80 modulation, we administered Ad-shTRAP80 to the liver via tail vein injection (Supplemental Figure 5). Five days after Ad-shTRAP80 injection, GW3965 was administered for 3 days. Surprisingly, we found that Ad-shTRAP80 prevented GW3965-induced hepatic steatosis (Figure 5A) and significantly reduced hepatic triglyceride accumulation (Figure 5B). In addition, Ad-shTRAP80 reduced the GW3965-induced increase in serum triglyceride levels (Figure 5C). Separation of serum lipoprotein classes by fast protein liquid chromatography (FPLC) indicated that the decrease of serum triglycerides in Ad-shTRAP80administered mice treated with GW3965 was attributed to a decrease of triglycerides in the VLDL fraction (Figure 5D). We then determined the effects of hepatic TRAP80 knockdown on the RCT process. The increases in both biliary cholesterol output and fecal neutral sterol loss by GW3965 were not affected by TRAP80 knockdown (Figure 5, E and F). In addition, we also observed an increase in HDL cholesterol by GW3965 in the mice injected with Ad-shTRAP80 (Figure 5G). The T0901317 treatment of Ad-shTRAP80-injected mice did not increase hepatic lipogenesis or hypertriglyceridemia, either (Supplemental Figure
6), whereas the level of anti-atherogenic HDL cholesterol was still increased by T0901317 under hepatic TRAP80-knockdown conditions (Supplemental Figure 6F). We also examined the effects of TRAP80 knockdown on the lipoprotein cholesterol and triglyceride profile in $\mathrm{Apoe}^{--}$mice, an experimental animal model of atherosclerosis, and found that the increase in VLDL cholesterol and triglycerides by GW3965 was diminished in Ad-shTRAP80injected $\mathrm{Apoe}^{-/-}$mice (Supplemental Figure 7). These results suggest that the detrimental effects of LXR activation could be selectively controlled by TRAP80 modulation.

Ad-shTRAP80 selectively inhibits lipogenic gene expression in the liver, but does not affect the expression of RCT-related genes or antiinflammatory effects in macrophages. In line with the serum lipid profile, the GW3965- and T0901317-induced transcription of lipogenic genes (Srebp-1c, Fas, and Scd1) was repressed by AdshTRAP80 expression in the liver (Figure 6A and Supplemental Figure 8A). On the other hand, Ad-shTRAP80 did not decrease GW3965- or T0901317-induced transcription of RCT-related genes (Abca1, Abcg5, and Abcg8) (Figure 6B and Supplemental Figure 8B). Recently, inducible degrader of low-density lipoprotein receptor ( $I d o l)$ was reported as a new LXR target gene that regulates hepatic cholesterol uptake by ubiquitination of the LDL 

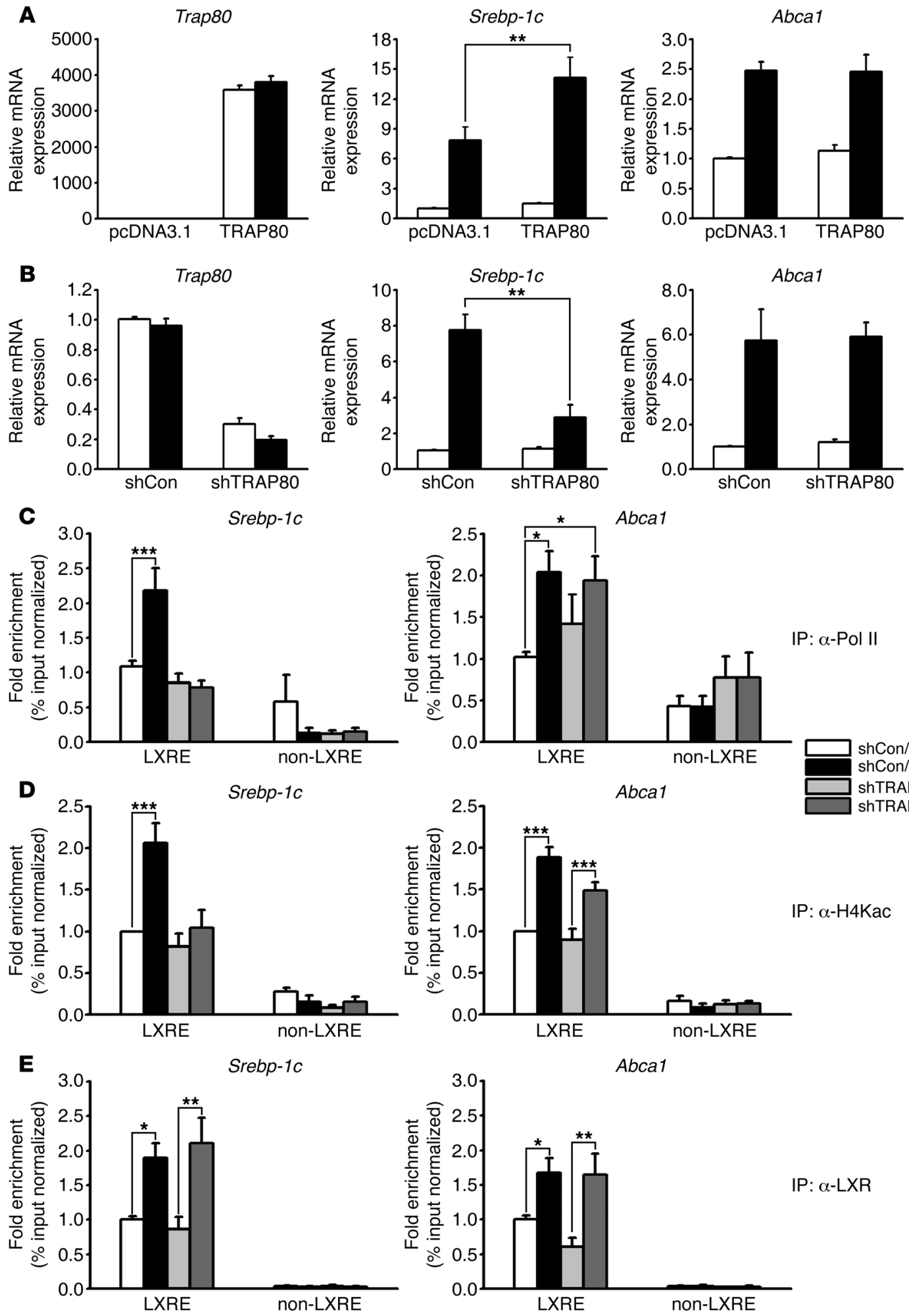

Figure 4. TRAP80 regulates Srebp-1c transcript levels by recruiting RNA Pol II. (A and B) Changes in Srebp-1c and Abca1 mRNA levels following TRAP80 overexpression (A, $n=5)$ or infection with Ad-shTRAP80 (B, $n=6)$ in mouse primary hepatocytes. (C-E) ChIP assays were performed with anti-Pol II (C, $n=5)$, anti-H4Kac $(\mathbf{D}, n=5)$, or anti-LXR $\alpha$ antibodies $(E, n=4)$ in mouse primary hepatocytes infected with Ad-shTRAP80. Abundance of each protein bound to Srebp-1c or Abca1 promoters was determined by qPCR. Enrichment levels are expressed as the fold enrichment relative to the enrichment observed in controls (shCon/DMSO). Data are presented as the mean \pm SEM. T1317, T0901317. ${ }^{*} P<0.05,{ }^{*} P<0.01$, and ${ }^{* * *} P<0.001$ by 1 -way ANOVA. 
A

shCon

GW3965
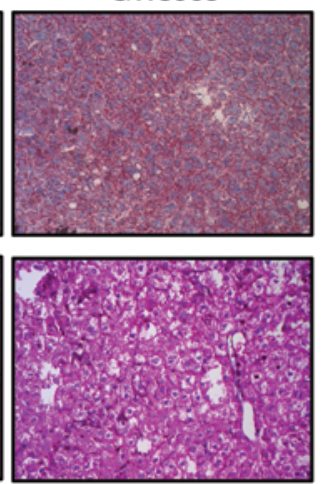

C

B

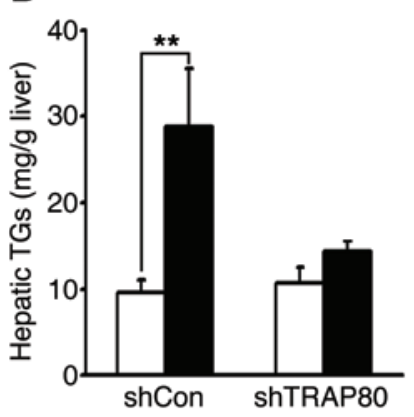

E

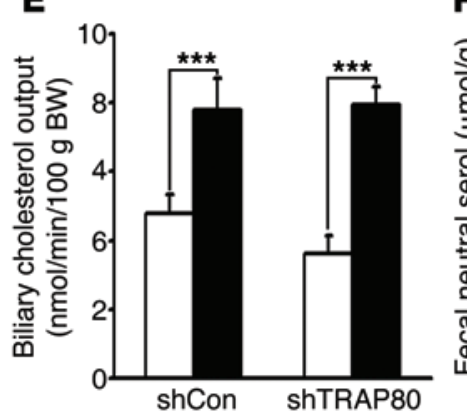

Vehicle

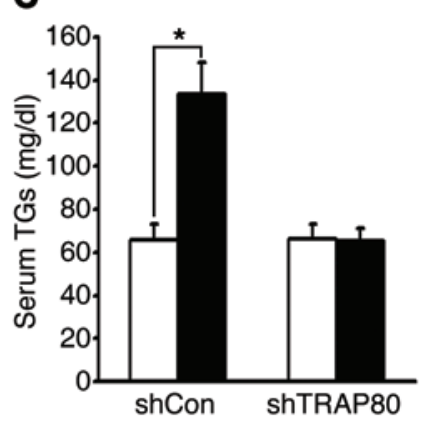

D
ShTRAP80

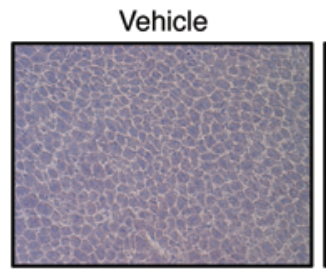

GW3965
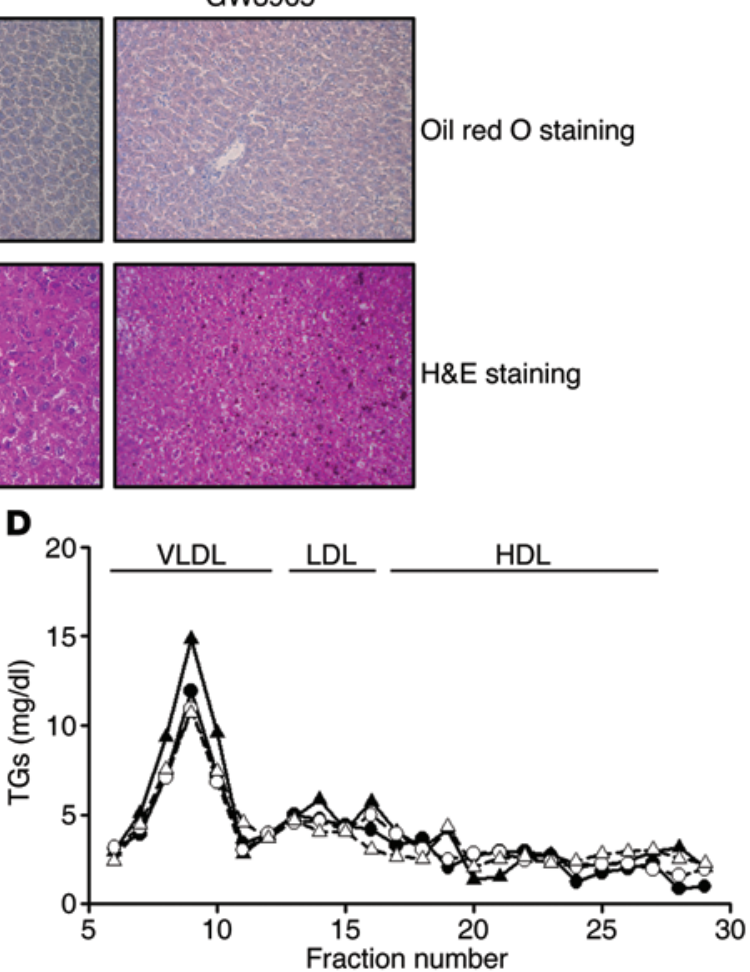

G

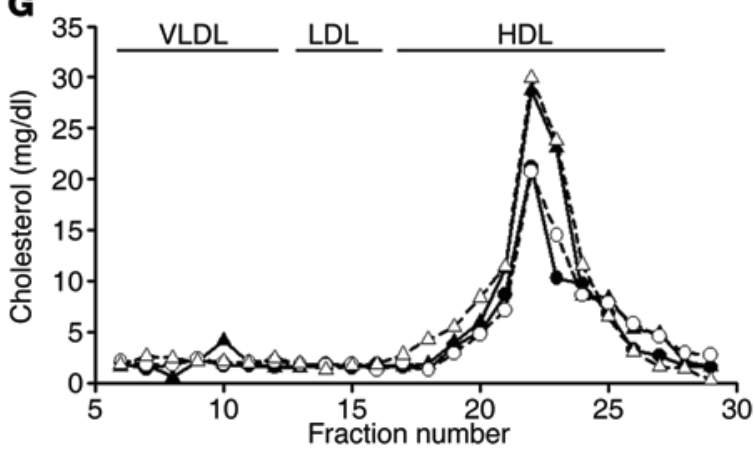

$\begin{array}{ll}\longrightarrow-\text { shCon/vehicle } & - \text { shCon/GW3965 } \\ -\infty-\text { shTRAP80/vehicle } & -- \text { shTRAP80/GW3965 }\end{array}$

Figure 5. Knockdown of hepatic TRAP80 ameliorates GW3965-induced fatty liver and an aberrant serum lipid profile. (A) Representative images of H\&E and oil red O-stained liver of Ad-shTRAP80-injected mice after administration of $50 \mathrm{mg} / \mathrm{kg}$ CW3965 for 3 days. Original magnification, $\times 100$. (B-D) Changes in hepatic triglyceride (B) and serum triglyceride (C and $\mathbf{D})$ levels in mice injected with Ad-shTRAP80 via the tail vein ( $n=5$ for each group). (E-G) Effects of Ad-shTRAP80 on GW3965-mediated RCT. Biliary cholesterol output (E), fecal neutral sterol (F), and HDL cholesterol (G) were measured ( $n=5$ for each group). The lipoprotein profile of mouse serum was analyzed by FPLC ( $\mathbf{D}$ and $\mathbf{G})$. Data are presented as the mean \pm SEM. TC, triglyceride. ${ }^{*} P<0.05$, ${ }^{* *} P<0.01$, and ${ }^{* *} P<0.001$, by 2 -tailed Student's $t$ test.

receptor (LDLR) (29). The IDOL-mediated inhibition of cholesterol uptake is independent of and complementary to the SREBP pathway (30). Interestingly, we found that the increases in Idol expression by GW3965 or T0901317 were differently affected by TRAP80 knockdown (Figure 6B and Supplemental Figure 8B). Meanwhile, macrophages are the primary cell type that becomes overloaded with cholesterol in atherosclerotic lesions and thus mediates the activation of the inflammatory pathway $(15,31)$. We found that the T0901317-dependent transcription of RCTrelated genes in mouse peritoneal macrophages was not affected by Ad-shTRAP80 (Figure 6C). In contrast, the LXR-dependent increases of lipogenic genes were repressed in Ad-shTRAP80infected macrophages (Figure 6D). Importantly, the inhibitory effects of T0901317 on the inflammatory gene response to LPS were reproduced in mouse peritoneal macrophages, even under TRAP80-knockdown conditions (Figure 6E). Taken together, these results suggest that TRAP80 selectively regulates the lipogenic activity of LXR, but not RCT or the antiinflammatory effects induced by LXR activation. On the basis of the results described herein, we propose a model for selective activation of the RCT process by simultaneous TRAP80 knockdown and treatment with LXR ligand (Figure 7). 

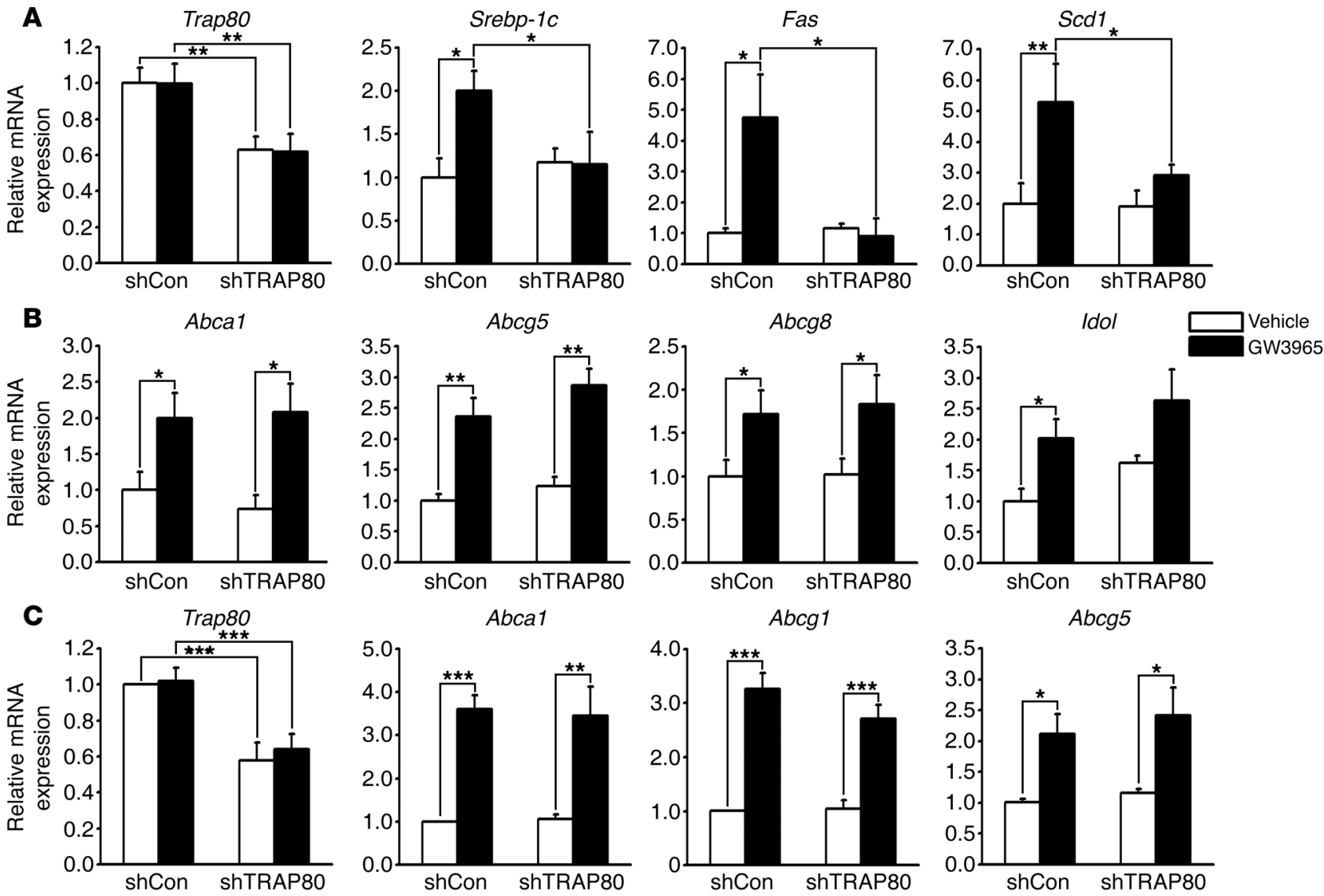

Abca1
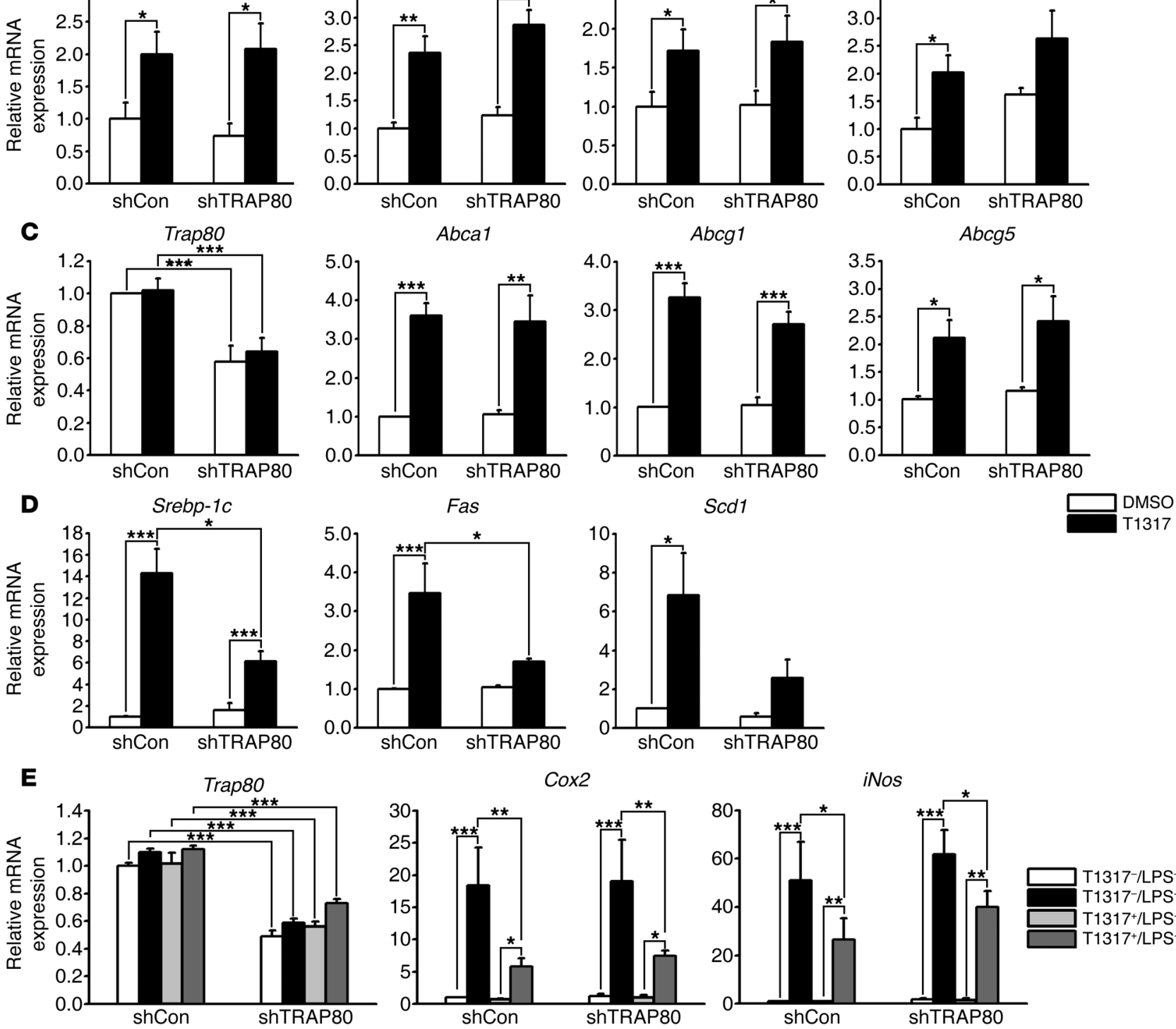

Figure 6. Effects of TRAP80 on the expression of metabolic and inflammatory genes in the liver and peritoneal macrophages. (A and B) Effects of TRAP80 knockdown on the transcript levels of lipogenesis- (A) or RCT-related genes (B) in the livers of mice treated with GW3965 ( $n=5$ for each group). mRNA levels were measured by qRT-PCR. (C and $\mathbf{D})$ Effects of Ad-shTRAP80 on the transcript levels of RCT- (C) or lipogenesis-related genes (D) in mouse peritoneal macrophages treated with DMSO or $1 \mu \mathrm{M}$ T0901317 $(n=4)$. (E) Effects of Ad-shTRAP80 on the transcript levels of inflammation-related genes in mouse peritoneal macrophages $(n=4)$. Macrophages were pretreated with $1 \mu \mathrm{M}$ T0901317 for 18 hours and then challenged with $100 \mathrm{ng} / \mathrm{ml} \mathrm{LPS}$ for another 24 hours. Data are presented as the mean \pm SEM. ${ }^{*} P<0.05,{ }^{* *} P<0.01$, and ${ }^{* *} P<0.001$, by 1 -way ANOVA.

\section{Discussion}

In the present study, we identified LXR $\alpha$-interacting nuclear proteins in HepG2 cells in an effort to elucidate the lipogenic processes mediated by LXR in the liver. There are 2 subtypes of LXR: LXR $\alpha$ and LXR $\beta$. No obvious phenotype has yet been attributed to Lxrbknockout mice. However, Lxra-knockout mice exhibited decreased serum triglyceride levels as well as decreased hepatic mRNA levels of multiple enzymes that participate in fatty acid synthesis, 


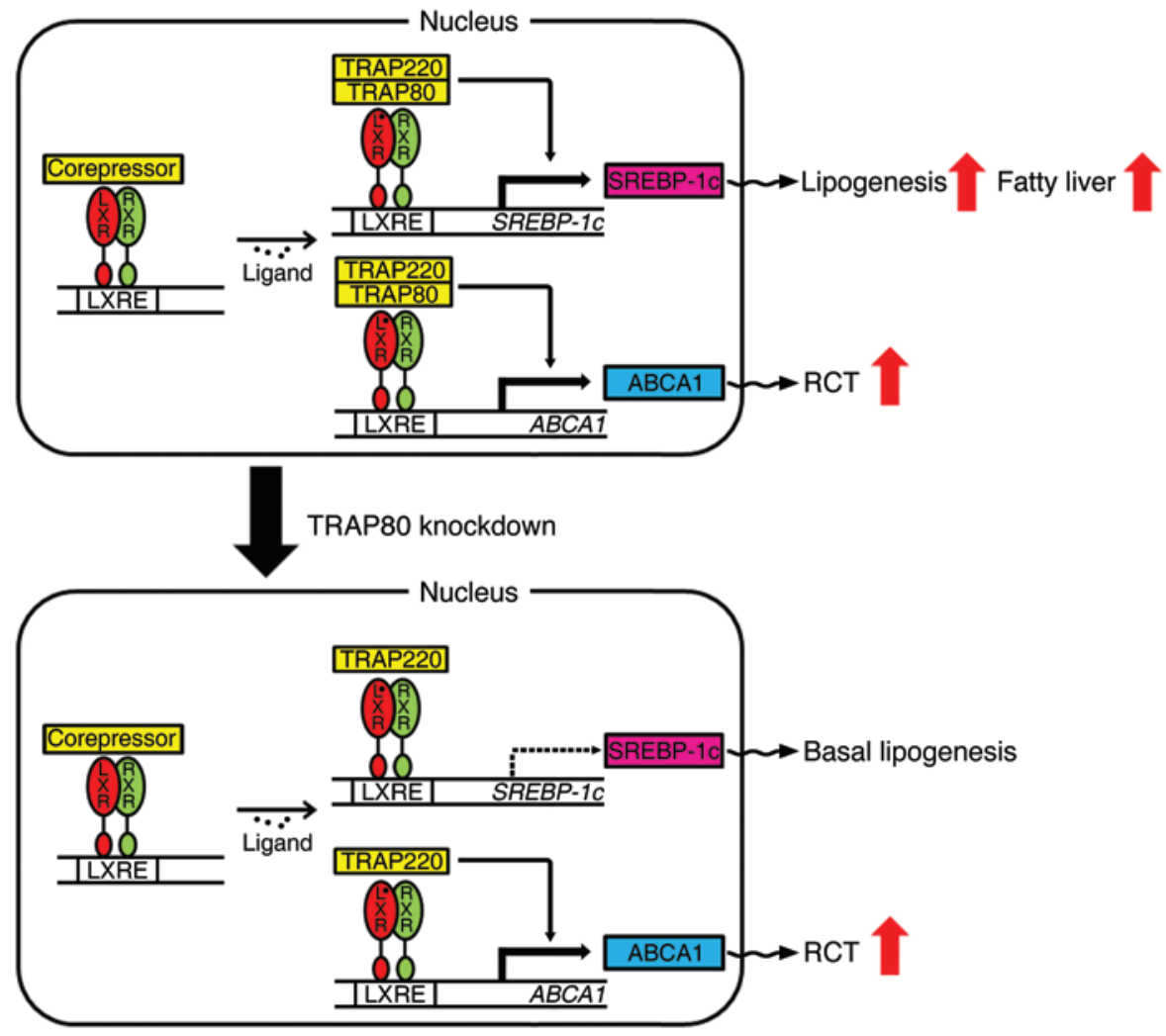

Figure 7. Working model for selective activation of RCT-related genes by simultaneous TRAP80 knockdown and treatment with LXR ligand. TRAP80 is essential for the activation of lipogenic genes including SREBP-1c, but not for RCT-related genes, such as $A B C A 1$. Therefore, under conditions of TRAP80 knockdown, LXR ligand treatment selectively activates RCT-related genes. mone receptors as well as other classes of transcription factors, most studies focus on TRAP220/DRIP205, because it is thought to form an interface between the nuclear receptor and the TRAP complex and interact with nuclear receptors in a ligand-dependent manner (37). Consequently, very little is known about the other subunits of the TRAP complex, including TRAP80. TRAP80 is a 651amino acid protein and is equivalent to the p78 component of the mouse Mediator (38). Our study shows that TRAP80 interacts with LXR $\alpha$ LBD in a ligand-dependent manner in nuclear extract-binding experiments and in a ligand-independent manner in 2-component GST pull-down assays. The same interaction pattern was seen between vitamin $\mathrm{D}$ receptor-LBD (VDR-LBD) and TRAP80/DRIP77 (36). This suggests that the ligand-dependent interactions between TRAP80 and LXR and VDR require nuclear component(s) other than TRAP80 and LXR or VDR alone. Interestingly, although full-length dTRAP80 of Drosophila interacts with retinoid X receptor (RXR) LBD only in the presence of the RXR ligand, 9-cis retinoic acid, a fragment of dTRAP80 possesses a sticky region that interacts with RXR LBD, regardless of the presence of ligand (39).

including FAS (32). These findings suggest that LXR $\alpha$ is primarily responsible for hepatic triglyceride accumulation and hypertriglyceridemia, effects that have hampered the development and use of LXR agonists as therapeutic agents (13). The adverse lipogenic effects of LXR $\alpha$ are in part mediated by LXR-induced transcription of $S R E B P-1 c$, a transcription factor that also induces the transcription of numerous lipogenic genes (33-35). To circumvent the adverse effect of LXR agonists, it is necessary to understand the mechanism by which SREBP-1c is activated by LXR $\alpha$ and identify the proteins involved in this activation process.

In this regard, we report for the first time to our knowledge the role of TRAP80 in the transcriptional regulation of $S R E B P-1 c$ by LXR $\alpha$. TRAP80 was first isolated as a component of the thyroid hormone receptor-associated protein (TRAP), which was initially identified as a large multimeric complex that copurifies with the thyroid hormone receptor (TR) from HeLa cells and distinctly increases TR-mediated transcription in vitro (23). Later, TRAP80 was also shown to interact directly with p53 and VP16 activation domains (22). TRAP80, also called DRIP77, ARC77, or CRSP77, is also identified as a subunit of vitamin D receptor-interacting protein (DRIP), activator-recruited cofactor (ARC), or cofactor required for Sp1 activation (CRSP), respectively $(24,26,36)$. ARC can be purified by affinity chromatography using the SREBP- $1 \alpha$ activation domain and has been shown to interact directly with VP16 and the NF-кB p65 subunit (26). Although more recent studies implicate TRAP as a coactivator of a wide range of nuclear hor-
Here, we purified 4 coactivators with LXR $\alpha$ in the presence of ligand from hepatoma HepG2 cells and from HEK 293T cells transfected with FLAG-LXR $\alpha$. Two of the coactivators, TRAP220 and TRAP80, interacted with LXR $\alpha$ directly and enhanced ligand-dependent transcription of the synthetic LXRE-driven reporter gene. However, while TRAP220 activated the transcription of both SREBP-1c and ABCA1, TRAP80 selectively activated SREBP-1c, but not ABCA1, although both are LXR target genes. This specificity of TRAP80 is attributed to selective recruitment to the $S R E B P-1 c$ promoter, but not to the $A B C A 1$ promoter. The ligand-stimulated recruitment of TRAP80 to the SREBP-1c LXRE was completely dependent on LXR $\alpha$. On the other hand, it is noteworthy that LXR $\alpha$-specific knockdown did not block TRAP220 recruitment to the $A B C A 1$ promoter, while it completely blunted ligand-dependent recruitment of both TRAP220 and TRAP80 to the SREBP-1c promoter. Based on these observations, we hypothesized that LXR $\alpha$ plays a major role in T0901317- or GW3965dependent activation of the SREBP-1c gene and recruitment of relevant coactivators to the LXRE region of $S R E B P-1 c$, while LXR $\beta$ has a main role in the LXR ligand-dependent activation of the $A B C A 1$ gene via the recruitment of TRAP220.

The ChIP assay using anti-RNA Pol II and -H4KAc antibodies showed that TRAP80 could facilitate the recruitment of RNA Pol II via chromatin remodeling through acetylation of $\mathrm{H} 4$ near the LXRE region of the Srebp-1c gene. However, we observed that LXR was recruited to the LXRE regions of both Srebp-1c and Abca1 
genes, irrespective of TRAP80 expression. This suggests that TRAP80 stimulates SREBP-1c transcription through the increase in RNA Pol II recruitment and histone acetylation, at least in part, but not through the enhancement of LXR binding. Consequently, Ad-shTRAP80 selectively decreased lipogenic gene expression, but did not affect the expression of RCT-related genes or the antiinflammatory effects of the LXR ligand. Meanwhile, IDOL is the E3 ubiquitin ligase that stimulates LDLR degradation and thus inhibits cholesterol uptake. In our study, Idol expression was differently regulated by Ad-shTRAP80, depending on the LXR ligands. The meaning of this differential regulation of IDOL by Ad-shTRAP80 in the context of different LXR ligands remains to be determined.

These differential effects of TRAP80 on LXR target gene expression resulted in alleviation of fatty liver and potentiation of RCT activity in the combinatorial treatment with LXR agonist and shTRAP80. In terms of RCT, we measured the expression of ABC transporters (ABCA1, ABCG1, ABCG5, and ABCG8) and the levels of biliary cholesterol and fecal sterol. RCT is a multistep process that occurs across several tissues including macrophages, liver, and intestine. Moreover, the $\mathrm{ABC}$ transporters are involved in the overall RCT process, from macrophages to feces, not just in cholesterol efflux from macrophages. Thus, we investigated the very last steps in RCT - biliary cholesterol secretion and fecal sterol loss - to evaluate the effect of TRAP80 on LXR-stimulated RCT.

This study suggests that the manipulation of TRAP80 represents a mechanism by which SREBP-1c-mediated lipogenesis can be selectively repressed while preserving RCT stimulation by LXR $\alpha$. Therefore, TRAP80 could be a promising target not only for the treatment of fatty liver, but also for the development of LXR agonists that effectively prevent atherosclerosis. In addition, the mechanism of coactivator selectivity described here might be used to modulate the specific expression of other key regulatory proteins responsible for the pathogenesis of a diverse array of diseases.

\section{Methods}

GST pull-down assays. GST pull-down assays were performed as described previously (25). Prior to incubation with the HepG2 nuclear extracts, glutathione-sepharose bead-immobilized GST and GSTLXR $\alpha$ LBD were normalized to equimolarity by quantitation using SDSPAGE. After binding (20 mM HEPES [pH 7.6], 20\% glycerol, $0.2 \mathrm{mM}$ EDTA, $180 \mathrm{mM} \mathrm{KCl}, 1 \mathrm{mM}$ DTT, 0.05\% nonidet P-40, $0.5 \mathrm{mM}$ PMSF, $10 \mu \mathrm{M}$ T0901317) and washing (20 mM HEPES [pH 7.6], 20\% glycerol, 0.2 mM EDTA, $180 \mathrm{mM} \mathrm{KCl,} 1 \mathrm{mM}$ DTT, 0.1\% nonidet P-40, $0.5 \mathrm{mM}$ PMSF), samples were eluted with $0.2 \%$ sarkosyl in wash buffer, resolved by $4 \%$ to $20 \%$ SDS-PAGE (Bio-Rad), and analyzed either by staining with Silver Stain Plus (Bio-Rad) or by immunoblot analysis.

Identification of $L X R \alpha L B D$-interacting proteins by mass spectrometry. LXR $\alpha$ LBD-interacting proteins in silver-stained gel slices were subjected to in-gel trypsin digestion. Extracted peptides were desalted using a ZipTip (EMD Millipore), resuspended in matrix, and subjected to MALDI-TOF analysis using a Voyager Biospectrometry system (Applied Biosystems) to yield a peptide mass fingerprint. Data were processed with the Mascot program (www.matrixscience.com).

IP and immunoblot analyses. HEK293T cells were treated with $1 \mu \mathrm{M}$ T0901317 for 2 hours, washed in PBS, and lysed on ice for 10 minutes in lysis buffer consisting of $1 \%$ NP-40, 10\% glycerol, and 50 $\mathrm{mM}$ Tris- $\mathrm{Cl}$ (pH 7.4). IP was performed by adding $6 \mu \mathrm{g}$ anti-FLAG antibody (F7425; Sigma-Aldrich) and incubating overnight at $4^{\circ} \mathrm{C}$ in IP buffer $(180 \mathrm{mM} \mathrm{KCl}, 1 \% \mathrm{NP}-40,10 \%$ glycerol, and $50 \mathrm{mM}$ Tris- $\mathrm{Cl}$ [pH 7.4]). Immune complexes were precipitated with protein $\mathrm{G}$-agarose (sc-2002; Santa Cruz Biotechnology Inc.), washed with IP buffer, and resuspended in reduced sample buffer. Proteins were then separated by SDS-PAGE, transferred onto PVDF membranes (Qbiogene), and detected with antibodies against TRAP230 (sc-5374), TRAP220 (sc-5334), CRSP130 (sc-12454), TRAP80 (ARP34192_P050; Aviva Systems Biology), $\beta$-actin (A5316; Sigma-Aldrich), and FLAG.

Reporter plasmid construction and luciferase reporter assay. Promoter-reporter constructs, including SREBP-1c-Luc, SREBP-1c (mtLX$R E$ )-Luc, ABCA1-Luc, and ABCA1 (mtLXRE)-Luc, were generated using a PCR-cloning strategy. The PCR primer sequences are listed in Supplemental Table 2. The TRAP80 expression construct was provided by Jae U. Jung (University of Southern California, Los Angeles, California, USA). The TRAP230 and CRSP130 were provided by Robert Roeder (Rockefeller University, New York, New York, USA) and Mononari Uesugi (Kyoto University, Kyoto, Japan), respectively. The reporter gene and $\beta$-gal constructs were cotransfected into HepG2 cells with or without $\mathrm{LXR} \alpha, \mathrm{RXR} \alpha$, TRAP230, TRAP220, CRSP130, and TRAP80 expression constructs using Lipofectamine 2000 (Invitrogen) according to the manufacturer's protocol. Eighteen to 24 hours after transfection, cells were treated with T0901317 or GW3965 for 24 hours. Cells were then collected for the measurement of luciferase and $\beta$-gal activity. Luciferase activity was measured using a Luminometer Centro LB 960 (Berthold) and normalized against $\beta$-gal activity.

ChIP assay. ChIP assays were performed as described previously, with minor modifications (40). Briefly, cells were treated with $1 \%$ formaldehyde for 20 minutes at room temperature. Next, cells were washed with cold PBS and resuspended in lysis buffer containing 50 mM Tris-HCl (pH 8.0), 1\% SDS, and 10 mM EDTA, followed by incubation for 30 minutes at $4^{\circ} \mathrm{C}$. Soluble chromatin from cells was prepared by sonication and immunoprecipitated with antibodies against TRAP220 (sc-5334; Santa Cruz Biotechnology Inc.), TRAP80 (sc12453; Santa Cruz Biotechnology Inc.), RNA polymerase II (sc-900X; Santa Cruz Biotechnology Inc.), H4Kac (06-598; EMD Millipore), and LXR (sc-13068; Santa Cruz Biotechnology Inc.). The final DNA extracts were amplified by qPCR using primers specific for the LXRE and non-LXRE regions of Srebp-1c and Abcal (Supplemental Table 3). The ChIP-qPCR data were normalized to input DNA sample and expressed as a fold change.

Generation of TRAP8O Ad-shRNA. To construct Ad-shTRAP80, shRNA-coding oligonucleotides were synthesized. For insertion of the shRNA sequence into the adenoviral shuttle vector pRNAT-H1.1/ Adeno (SD1219; GenScript Inc.), MluI/HindIII restriction sequences and a T5 terminal sequence were included in the shRNA-encoding oligonucleotide (Supplemental Table 2). The synthesized oligonucleotides were annealed and inserted into pRNAT-H1.1/Adeno. The shRNA-containing shuttle vector was then linearized using PmeI, and the linearized pRNAT1.1/Adeno was recombined with the pAdEasy-1 vector by in vivo homologous recombination in the $E$. coli strain BJ5183. The resulting DNA was digested with PacI and transfected into Ad-293 cells using Lipofectamine 2000. After 2 to 3 days, cGFP expression was observed in Ad-293 cells by a fluorescence microscopy. Cells were harvested and lysed by the freeze-thaw method. Adenovirus-containing supernatant was obtained by centrifugation and used to infect the Ad-293 cells. This process was repeated 3 times. 
Supernatants obtained from each step were stored at $-70^{\circ} \mathrm{C}$. For the infection of mice and mouse primary hepatocytes, Ad-293 cells were infected with crude stock virus, lysed by freezing and thawing after 24 to 48 hours, and the viruses were purified using $\mathrm{CsCl}_{2}$ gradients.

RNA isolation and $q R T-P C R$. Total RNA was purified from HepG2 cells, mouse primary hepatocytes, peritoneal macrophages, or adenovirus-infected livers using TRIzol (Invitrogen) and reverse transcribed using MMLV reverse transcriptase (Promega) according to the manufacturer's instructions. Quantitative gene expression analysis was performed with the LightCycler 480 System (Roche) using SYBR Green PCR Master Mix (Invitrogen). The PCR primers used in this study are listed in Supplemental Table 4. RPS29 (ribosomal protein S29) was used as an internal control.

Mice. Six-to 8-week-old male C57BL/6N mice were purchased from Orient Bio Inc. Six-week-old male Apoe-deficient spontaneously hyper-

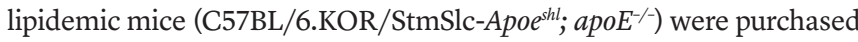
from Central Lab Animal Inc. Mice were maintained in a controlled environment with a 12-hour light/12-hour dark cycle and ad libitum access to water and standard rodent chow.

Generation of hepatic TRAP8O-knockdown mice and induction of fatty liver by LXR activation. Recombinant adenovirus Ad-shTRAP80 or Ad-shCon was delivered to C57BL/6N or Apoe $e^{-/}$mice by tail vein injection at a dose of $1 \times 10^{11}$ particles per $20 \mathrm{~g}$ of body weight. On the fifth day after virus injection, mice were treated with $50 \mathrm{mg} / \mathrm{kg}$ T0901317 or GW3965, administered daily by oral gavage for 3 days. T0901317 or GW3965 was dissolved in 0.5\% methylcellulose (M0262; SigmaAldrich) to a final concentration of $3.33 \mathrm{mg} / \mathrm{ml}$ and administered at a dose of $0.3 \mathrm{ml}$ per $20 \mathrm{~g}$ of body weight. On the eighth day after virus injection, blood was obtained by heart puncture. Livers were harvested, snap-frozen in liquid nitrogen, and stored at $-70^{\circ} \mathrm{C}$ until analysis.

Metabolic analyses of mouse serum and liver samples. Serum triglycerides, total cholesterol, HDL-cholesterol, and LDL/VLDL cholesterol were measured in serum samples using analysis kits purchased from Asan Pharmaceutical (total and HDL cholesterol), SigmaAldrich (serum triglycerides), and BioVision (LDL/VLDL cholesterol). The biochemical separation of HDL and LDL/VLDL was achieved using LDL/VLDL precipitation buffer (K613-100-2; BioVision). The supernatant is an HDL fraction, and the precipitate is an LDL/VLDL fraction. To determine lipoprotein lipid distribution, pooled serum $(50 \mu \mathrm{l})$ from mice was fractionated by FPLC gel filtration (ÄKTA System; GE Healthcare) on a Superose 6 3.2/300 column (29-0362-26; GE Healthcare) at a flow rate of $40 \mu \mathrm{l} /$ minute. The cholesterol and triglyceride concentrations in the FPLC fractions were determined enzymatically using assay kits (K623-100; BioVision, for cholesterol; AM157S-K; Asan Pharmaceutical, for triglycerides). For the measurement of hepatic triglycerides, lipid components were extracted from livers using chloroform containing 1\% Triton X-100. After the chloroform was evaporated, hepatic triglycerides were dissolved in Triton X-100 and assayed using an analysis kit (TR0100; Sigma-Aldrich).

Measurement of biliary cholesterol output and fecal neutral sterol. For bile collection, mice were anesthetized, and the peritoneal cavity was opened. The common bile duct was tied off by silk ligature, and the gallbladder was nicked and cannulated with a PE-10 tube (427401; $\mathrm{BD})$. Bile samples were collected for 30 minutes, while the body temperature was maintained by placing the animal under a heat lamp. Cholesterol in bile was determined by an enzymatic method using total cholesterol assay kits (AM202-K; Asan Pharmaceutical). For the measurement of fecal neutral sterol, feces were collected on the last 2 days before sacrificing the mice. Feces samples were dried, weighed, and ground, and $5 \alpha$-cholestane was added as an internal standard. The sample was then saponified and extracted using cyclohexane. After extraction, neutral sterols were converted to trimethylsilyl-ether (TMS-ether) derivatives for gas-liquid chromatographic analysis.

Statistics. Results are expressed as the mean \pm SEM. Statistical significance was assessed using an unpaired, 2-tailed Student's $t$ test or 1-way ANOVA. Differences with $P$ values of less than 0.05 were considered statistically significant.

Study approval. All animals were maintained and handled according to protocols approved by the IACUC of the Asan Institute for Life Sciences.

\section{Acknowledgments}

We are grateful to Robert Roeder for providing TRAP230; Mononari Uesugi for providing DRIP130 (CRSP130); and Jae U. Jung for providing TRAP80 (CRSP77). We also thank Han Choe (Department of Physiology, University of Ulsan College of Medicine) and Hyun Ju Yoo (Metabolomics Core, Asan Institute for Life Sciences) for their help with FPLC lipoprotein analysis and GC analysis of fecal neutral sterol, respectively. This study was supported by grants from the Korea Healthcare Technology R\&D Project (A084335); the Ministry for Health, Welfare \& Family Affairs of the Republic of Korea; the National Research Foundation of Korea funded by the Korean Government (NRF-2006-2005402); and by intramural grants 2009-352 and 2010-352 (to S.W. Kim) from the Asan Institute for Life Sciences.

Address correspondence to: Seung-Whan Kim, 88, Olympic-Ro 43-gil, Songpa-Gu, Seoul 138-736, Republic of Korea. Phone: 822.3010.4297; E-mail: swkim7@amc.seoul.kr.

Geun Hyang Kim's present address is: Sanford-Burnham Medical Research Institute at Lake Nona, Orlando, Florida, USA.
1. Zelcer N, Tontonoz P. Liver X receptors as integrators of metabolic and inflammatory signaling. JClin Invest. 2006;116(3):607-614.

2. Janowski BA, Willy PJ, Devi TR, Falck JR, Mangelsdorf DJ. An oxysterol signalling pathway mediated by the nuclear receptor LXR $\alpha$. Nature. 1996;383(6602):728-731.

3. Rosenfeld MG, Glass CK. Coregulator codes of transcriptional regulation by nuclear receptors. J Biol Chem . 2001;276(40):36865-36868.

4. McKenna NJ, O’Malley BW. Combinatorial con- trol of gene expression by nuclear receptors and coregulators. Cell. 2002;108(4):465-474.

5. Aoyagi S, Archer TK. Dynamics of coactivator recruitment and chromatin modifications during nuclear receptor mediated transcription. Mol Cell Endocrinol. 2008;280(1-2):1-5.

6. Han SJ, DeMayo FJ, Xu J, Tsai SY, Tsai M-J, O’Malley BW. Steroid receptor coactivator (SRC)-1 and SRC-3 differentially modulate tissue-specific activation functions of the progesterone receptor. Mol Endocrinol. 2006;20(1):45-55.
7. Feige JN, Auwerx J. Transcriptional coregulators in the control of energy homeostasis. Trends Cell Biol. 2007;17(6):292-301.

8. Kim SW, et al. Activating signal cointegrator 2 required for liver lipid metabolism mediated by liver X receptors in mice. Mol Cell Biol. 2003;23(10):3583-3592.

9. $\mathrm{Kim} \mathrm{GH}$, et al. Characterization of ASC-2 as an antiatherogenic transcriptional coactivator of liver X receptors in macrophages. Mol Endocrinol. 2009;23(7):966-974. 
10. Roger VL, et al. Heart disease and stroke statistics-2012 update: a report from the American Heart Association. Circulation. 2012;125(1):e2-e220.

11. Joseph SB, Castrillo A, Laffitte BA, Mangelsdorf DJ, Tontonoz P. Reciprocal regulation of inflammation and lipid metabolism by liver $\mathrm{X}$ receptors. Nat Med. 2003;9(2):213-219.

12. Naik SU, et al. Pharmacological activation of liver $\mathrm{X}$ receptors promotes reverse cholesterol transport in vivo. Circulation. 2006;113(1):90-97.

13. Lund EG, Menke JG, Sparrow CP. Liver X receptor agonists as potential therapeutic agents for dyslipidemia and atherosclerosis. Arterioscler Thromb Vasc Biol. 2003;23(7):1169-1177.

14. Zhang Y, et al. Liver LXR $\alpha$ expression is crucial for whole body cholesterol homeostasis and reverse cholesterol transport in mice. J Clin Invest. 2012;122(5):1688-1699.

15. Cuchel M, Rader DJ. Macrophage reverse cholesterol transport: Key to the regression of atherosclerosis? Circulation. 2006;113(21):2548-2555.

16. Terasaka N, et al. T-0901317, a synthetic liver X receptor ligand, inhibits development of atherosclerosis in LDL receptor-deficient mice. FEBS Lett. 2003;536(1-3):6-11.

17. Joseph SB, et al. Synthetic LXR ligand inhibits the development of atherosclerosis in mice. Proc Natl Acad Sci U S A. 2002;99(11):7604-7609.

18. Im S-S, Osborne TF. Liver X receptors in atherosclerosis and inflammation. Circ Res. 2011;108(8):996-1001.

19. Repa JJ, et al. Regulation of mouse sterol regulatory element-binding protein-1c gene (SREBP-1c) by oxysterol receptors, LXR $\alpha$ and LXR $\beta$. Genes Dev. 2000;14(22):2819-2830.

20. Schultz JR, et al. Role of LXRs in control of lipogenesis. Genes Dev. 2000;14(22):2831-2838.

21. Yoshikawa T, et al. Identification of liver X recep- tor-retinoid $\mathrm{X}$ receptor as an activator of the sterol regulatory element-binding protein $1 \mathrm{c}$ gene promoter. Mol Cell Biol. 2001;21(9):2991-3000.

22. Ito M, et al. Identity between TRAP and SMCC complexes indicates novel pathways for the function of nuclear receptors and diverse mammalian activators. Mol Cell. 1999;3(3):361-370.

23. Fondell JD, Ge H, Roeder RG. Ligand induction of a transcriptionally active thyroid hormone receptor coactivator complex. Proc Natl Acad Sci US A. 1996;93(16):8329-8333.

24. Ryu S, Zhou S, Ladurner AG, Tjian R. The transcriptional cofactor complex CRSP is required for activity of the enhancer-binding protein Sp1. Nature. 1999;397(6718):446-450.

25. Rachez C, et al. A novel protein complex that interacts with the vitamin D3 receptor in a ligand-dependent manner and enhances VDR transactivation in a cell-free system. Genes Dev. 1998;12(12):1787-1800.

26. Naar AM, Beaurang PA, Zhou S, Abraham S, Solomon W, Tjian R. Composite co-activator ARC mediates chromatin-directed transcriptional activation. Nature. 1999;398(6730):828-832.

27. Berger SL. Histone modifications in transcriptional regulation. Curr Opin Genet Dev. 2002;12(2):142-148.

28. Eberharter A, Becker PB. Histone acetylation: a switch between repressive and permissive chromatin. Second in review series on chromatin dynamics. EMBO Rep. 2002;3(3):224-229.

29. Zelcer N, Hong C, Boyadjian R, Tontonoz P. LXR regulates cholesterol uptake through Idol-dependent ubiquitination of the LDL receptor. Science. 2009;325(5936):100-104.

30. Zhang L, Reue K, Fong LG, Young SG, Tontonoz P. Feedback regulation of cholesterol uptake by the LXR-IDOL-LDLR axis. Arterioscler Thromb Vasc Biol. 2012;32(11):2541-2546.
31. Tabas I. Macrophage death and defective inflammation resolution in atherosclerosis. Nat Rev Immunol. 2010;10(1):36-46.

32. Peet DJ, et al. Cholesterol and bile acid metabolism are impaired in mice lacking the nuclear oxysterol receptor LXR $\alpha$. Cell. 1998;93(5):693-704.

33. Magana MM, Osborne TF. Two tandem binding sites for sterol regulatory element binding proteins are required for sterol regulation of fatty-acid synthase promoter. J Biol Chem. 1996;271(51):32689-32694.

34. Magana MM, Lin SS, Dooley KA, Osborne TF. Sterol regulation of acetyl coenzyme A carboxylase promoter requires two interdependent binding sites for sterol regulatory element binding proteins. J Lipid Res. 1997;38(8):1630-1638.

35. Foretz M, et al. ADD1/SREBP-1c is required in the activation of hepatic lipogenic gene expression by glucose. Mol Cell Biol. 1999;19(5):3760-3768.

36. Rachez C, et al. Ligand-dependent transcription activation by nuclear receptors requires the DRIP complex. Nature. 1999;398(6730):824-828.

37. Yuan CX, Ito M, Fondell JD, Fu ZY, Roeder RG. The TRAP220 component of a thyroid hormone receptor- associated protein (TRAP) coactivator complex interacts directly with nuclear receptors in a ligand-dependent fashion. Proc Natl Acad Sci US A. 1998;95(14):7939-7944.

38. Jiang YW, et al. Mammalian mediator of transcriptional regulation and its possible role as an end-point of signal transduction pathways. Proc Natl Acad Sci U S A. 1998;95(15):8538-8543.

39. Park JM, et al. Signal-induced transcriptional activation by Dif requires the dTRAP80 mediator module. Mol Cell Biol. 2003;23(4):1358-1367.

40. Orlando V, Strutt H, Paro R. Analysis of chromatin structure by in vivo formaldehyde cross-linking. Methods. 1997;11(2):205-214. 